allowed body checking; 120 players participated in more than one season.

Assessment of Risk Factors Exposure to policy that permits body checking (Edmonton 2015-17 and Calgary 2015-18) vs. policy disallowing body checking (Vancouver 2015-16, Edmonton 2016-17, and Calgary 2016-18).

Main Outcome Measurements All game-related ice hockeyrelated injuries were identified by a team safety designate. Suspected concussions were referred to a study sport medicine physician.

Results In divisions allowing body checking, there were 213 injuries [incidence rate $(I R)=12.96 / 1000$ player-hours; 95\% CI: 9.21-16.70] including 69 concussions ( $I R=4.20 / 1000$ player-hours; 95\% CI: 2.63-5.76). In divisions disallowing body checking, there were 40 injuries (IR $=5.13 / 1000$ playerhours; 95\% CI: 1.71-8.56) including 18 concussions (IR= 2.31/1000 player-hours; 95\% CI: 0.62-4.00). Using multilevel Poisson regression adjusted for cluster and several important covariates (year of play, player weight, previous injury/concussion history, position), policy disallowing body checking was associated with a lower rate of all injury [incidence rate ratio $[\mathrm{IRR}]=0.38$; 95\% CI: $0.24-0.60)$, and concussion $(\mathrm{RR}=0.49$; 95\% CI: $0.26-0.89$ ).

Conclusions Policy disallowing body checking reduced the rate of game-related injuries in Midget non-elite levels of ice hockey. This research should inform body checking policy change nationally.

\section{PERCEIVED BARRIERS AND FACILITATORS TOWARDS AN INJURY PREVENTION PROGRAM AMONG PROFESSIONAL MALE ICE HOCKEY PLAYERS AND STAFF MEMBERS}

${ }^{1}$ Romana Brunner, ${ }^{1}$ Mario Bizzini, ${ }^{1}$ Nicola Maffiuletti, ${ }^{2}$ Karin Niedermann. 'Human Performance Lab, Schulthess Clinic, Zurich, Switzerland; ' ${ }^{2}$ Department of Health, Institute of Physiotherapy, Zurich University of Applied Sciences, Winterthur, Switzerland

\subsection{6/bjsports-2021-IOC.43}

Background Lower extremity injury prevention programs are effective in team sport athletes.

Objective This study identifies barriers to and facilitators for an injury prevention program in professional male ice hockey teams among players and staff members.

Design Cross-sectional survey.

Setting Professional male ice hockey teams.

Participants Eight Swiss National League A (NLA) teams and their staff members were invited to participate in the study and voluntarily completed the questionnaire. Inclusion criteria were the ability to understand German or English and signed informed consent. Six teams completed the questionnaire (148 players and 11 staff members).

Main outcome measurements Frequency of ratings related to knowledge, attitude, adherence and habits were analysed. Binary logistic regression analysis was applied to predict a relationship between players' characteristics and positive attitude towards an injury prevention program.

Results Knowledge, perceived benefit and relevance of injury prevention as well as awareness of high risk of injuries in ice hockey were identified as important facilitators. Players' habit of exercise performance was identified as a barrier. Program understanding of staff members was identified as a facilitator and barrier. No significant relationships were observed between a high/low perceived benefit of an injury prevention program and age $(\mathrm{p}=0.85)$, nationality $(\mathrm{p}=0.53)$, level of education $(\mathrm{p}=0.63)$, National League experience $(\mathrm{p}=0.50)$ or occurrence of lower extremity injuries in the previous season $(\mathrm{p}=0.10)$.

Conclusion Identified barriers and facilitators should be addressed when developing and implementing an injury prevention program in a setting of professional ice hockey teams. An injury prevention program should be offered to and implemented in players of all ages, levels of education and experience in National League, irrespective of previous injuries.

\section{THE MECHANISMS OF ANTERIOR CRUCIATE LIGAMENT INJURIES IN MALE PROFESSIONAL FOOTBALL PLAYERS IN THE MIDDLE EAST: A SYSTEMATIC VIDEO ANALYSIS OF 15 CASES}

Raouf Nader Rekik, Roald Bahr, Flavio Cruz, Pieter D'Hooghe, Paul Read, Montassar Tabben, Karim Chamari. Aspetar (orthopedic and sport medicine hospital), Doha, Qatar

\subsection{6/bjsports-2021-IOC.44}

Background While effective programmes to prevent anterior cruciate ligament (ACL) injury have been established, it is important that programme design, consistent with the van Mechelen model, is based on the specific injury mechanisms, representative of the region and playing conditions. Presently, no such data exist for professional football players competing in the Middle East.

Objective To describe the mechanisms of ACL injury in male professional football players in the Qatar Star League (QSL) based on systematic video analysis.

Design Systematic video analysis of prospective injury surveillance data.

Setting Male professional football in Qatar.

Participants Fifteen professional male football players in the QSL, who sustained an ACL injury in a match during the seasons 2013/2014 through 2018/2019.

Assessment of injury mechanisms Injuries were recorded through the Aspetar Injury Surveillance Programme and high definition broadcast videos were analyzed (49 views; 34 slow motion). Five analysts independently described the injury mechanisms in detail (situation, behavior, biomechanical characteristics), using validated observational tools (Walden et al. 2015).

Results A knee valgus mechanism was observed in 10 cases (1 with direct contact to the knee, 3 with contact to other body part and 6 with no body contact), no valgus was observed in 2 cases (both direct knee contact), while 3 were unclear. Of the 12 non-contact/indirect-contact situations, we saw 4 main categories of injury situations (multiple combinations were possible): pressing $(n=6)$, tackling or to be tackled by another player $(n=4)$, blocking $(n=3)$ and screening $(n=2)$. In the 3 direct contact injuries, the player was tackling in 2 cases and being tackled by another player in 1 case.

Conclusions Only 20\% of match ACL injuries were contact injuries. Knee valgus was consistently observed, independent of the playing situation. Pressing was the main mechanism leading to injury. Landing after heading was not observed in any of these injuries. 